\title{
The Pseudoscalar Interaction and the Beta Spectrum of RaE
}

\author{
M. E. Rose AND R. K. OsBoRN \\ Oak Ridge National Laboratory, Oak Ridge, Tennessee \\ (Received September 14, 1953; revised manuscript received December 17, 1953)
}

\begin{abstract}
It is shown that the theory of forbidden beta transitions, as hitherto used, does not provide a correct treatment of the pseudoscalar interaction. This is demonstrated by the application of a canonical transformation to the Hamiltonian of interacting nucleons and leptons whereby all odd nuclear Dirac operators are eliminated from the theory. When this is done properly it is seen that the pseudoscalar interaction makes a contribution to the $\beta$-decay process only by virtue of the fact that the lepton covariants are not constant. The corresponding additional operators introduced in the other three interactions ( $S$ is pure even) are examined and it is shown that, except in one case, these make trivial corrections which would not be observed in practice. The exception occurs in second and higher forbidden transitions wherein the spin change (tensor rank, strictly speaking) is lower than the forbiddenness order. By virtue of present knowledge of the beta interaction these must be regarded as small correction terms. The possibility of calculating all nuclear matrix elements using nonrelativistic wave functions based on some coupling model is discussed. The results presented here also show that wherever $P$ and $T$ interactions interfere (spin change zero, firstforbidden transitions) it will be possible to obtain the ratio of coupling coefficients ( $g_{P} / g_{T}$ for example) by comparison with the observed spectral shapes. A method of reduction of the $\beta$-decay operators is described and it is pointed out that the same procedure is very convenient for obtaining the nuclear matrix elements. In this method the irreducible tensors are obtained automatically and the retardation expansion is a trivial operation performed at the end of the calculation rather than at the beginning. The correction factors for pseudoscalar $(P)$ as well as $P-T$ and $P-A$ mixtures are obtained and the fact that the shape of the correction factor is strongly modified as compared to the customary result is observed. The correction factor is now more strongly $Z$ dependent and this would perhaps account for the appearance of the $P$ interaction only for heavy elements. The results of this investigation are applied to the RaE spectrum. No fit with the assumption of zero spin for RaE can be obtained. It is suggested that the spin of RaE is unity, and other evidence pointing in this direction is cited.
\end{abstract}

\section{INTRODUCTION}

$T$ $\mathrm{HE}$ somewhat anomalous beta-spectrum of $\mathrm{RaE}$ has attracted considerable attention in recent years. It is a well-known fact that despite several attempts to account for the observed spectrum, using the well-established theory of forbidden beta transitions, ${ }^{1}$ no success could be claimed until the analysis of Petschek and Marshak ${ }^{2}$ appeared. From that work one would conclude that the RaE spectrum demands the presence of the pseudoscalar $(P)$ interaction in the betacoupling and in fact, a tensor-pseudoscalar (T-P) mixture seemed to suffice to account for the observed results. ${ }^{3}$

The assumption upon which the aforementioned analysis of Petschek and Marshak is based is the assignment of zero spin to the decaying state and an a priori reason for this assignment is based on the Nordheim rule for minimum spin when neutron and proton configurations belong to different Schmidt groups. The odd parity assignment of the shell model and the $f t$ value makes it quite certain that the transition is first forbidden and the fact that the spectrum shape is definitely not that characteristic of a $|\Delta J|=2$ transition limits the spin values to 0 or 1 . With the exclusion of the $A$ interaction by the results of the measurement of the

\footnotetext{
${ }^{1}$ E. J. Konopinski and G. E. Uhlenbeck, Phys. Rev. 60, 308 (1941), referred to as $\mathrm{KU}$.

${ }_{2}^{2}$ A. G. Petschek and R. E. Marshak, Phys. Rev. 85, 698 (1952), referred to as PM.

3 For example, L. M. Langer and H. C. Price, Jr., Phys. Rev. 76, 461 (1949).
}

$\mathrm{He}^{6}$ angular correlation ${ }^{4}$ and the shapes of other first forbidden spectra ${ }^{5}$ one is led to a $P-T$ mixture as the only possible explanation of the spectrum if the spin 0 assignment is adopted.

Nevertheless, this analysis of the RaE spectrum in terms of the $T-P$ mixture cannot be accepted, because the otherwise completely correct theory of forbidden beta transitions is not correct insofar as the pseudoscalar interaction is concerned. The reasons for this statement will be presented below in detail. At this point it suffices to say that the proper correction factor to be used for the $P$ interaction and for all interference terms with the $P$ interaction differs from those hitherto used in a major way. There are also other cases in which the correction factors are misrepresented in a nontrivial way but these arise in circumstances which are now known to be impractical. This remark will also be amplified in the following.

At this stage of the discussion we may indicate in what manner the customary treatment fails. The central point involved is that in all forbidden transitions wherein, in the conventional form of the theory, odd Dirac operators are involved, one cannot make the usual categorical statement that the nuclear matrix elements are certain numbers independent of the parameters describing the lepton field. Actually, in such operators the small component of the nuclear wave function is involved and this implies that the momentum

\footnotetext{
4 B. M. Rustad and S. L. Ruby, Phys. Rev. 89, 880 (1952).

5 H. M. Mahmoud and E. J. Konopinski, Phys. Rev. 85, 1266 (1952).
} 
of the decaying nucleon appears. Because of the pointcoupling of the beta interaction, this will introduce terms directly dependent on the momenta of the leptons (more exactly, the gradient operator acting on the lepton covariant is introduced). Because the lepton de Broglie wavelengths are so large compared to the nuclear radius, such terms are always rejected. This, it turns out, is completely justified in all but two cases and one of these is the pseudoscalar interaction. In this case, if one follows the usual treatment, there is no contribution from the pseudoscalar interaction at all. ${ }^{6}$ In this respect the pseudoscalar interaction is unique. In fact, the word "peculiar" may be an appropriate description of such interactions.

The validity of statements made above become apparent if one carries out a thorough reduction of the beta-decay problem to the nonrelativistic limit so far as the nucleon space is concerned. This is done in Sec. II in such a way that one can see exactly what is neglected. The error involved in such a step is exceedingly minute so that the beta-decay problem can be described in a greatly simplified representation which is virtually exact.

A particularly interesting advantage which accrues from this procedure is the possibility of calculating nuclear matrix elements in terms of nonrelativistic wave functions. The implication here is that one adopts a specific coupling model ( $j-j$ or $L-S$, for example). This representation of the beta-decay operators in even form may be compared to the usual representation where it is necessary to calculate matrix elements of odd operators; the usual representation therefore requires relativistic wave functions concerning which one must profess almost complete ignorance. While our change of representation is a formal step, it facilitates the application of what one does know concerning nuclear forces from a phenomenological point of view. In some cases, (for mirror nuclei, say) no detailed knowledge of nuclear forces is necessary, just as was the case for certain even operators. ${ }^{7}$

Finally, we mention the fact that initial attempts in the direction we have here indicated were made by others. ${ }^{8}$ However, these investigations did not lead to correct results because the reduction to the nonrelativistic case was not made consistently.

\section{THE CANONICAL TRANSFORMATION OF THE BETA-DECAY OPERATORS}

Since, as implied above, the difficulty in the conventional formalism lies in the occurrence of odd Dirac

\footnotetext{
${ }^{6}$ This statement is almost rigorous. There may be extremely small contributions; see Sec. II.

${ }^{7}$ As a preliminary step we have investigated the matrix elements of the (formerly) odd operators in the one particle $j-j$ coupling model, M. E. Rose and R. K. Osborn, following paper [Phys. Rev. 93, 1326 (1954)]

8 Ahrens, Feenberg, and Primakoff, Phys. Rev. 87, 663 (1952); T. Ahrens, Phys. Rev. 90, 974 (1953). Part of our results are contained in the results of the latter paper, but to obtain the proper correction factor large parts of Ahren's results are simply to be discarded.
}

operators, we eliminate these by a canonical transformation (FW). ${ }^{9}$ Alternatively, one can proceed by the method of elimination of small components in the nuclear wave function. However, this procedure is cumbersome to apply in the case of a many-particle nucleus and is also somewhat less desirable from a theoretical point of view. ${ }^{9}$

For the system of nuclear sources coupled to the lepton field the Hamiltonian is

where

$$
H=H_{N}+H_{\beta}+H_{l},
$$

$$
H_{N}=-\boldsymbol{\alpha} \cdot \mathbf{p}-\frac{\beta}{2}\left[M_{n}\left(1+\tau_{z}\right)+M_{p}\left(1-\tau_{z}\right)\right]+V
$$

is the nuclear Hamiltonian, and

$$
H_{\beta}=\sum_{X} g_{X} \Omega_{X}^{\mu}(N) \Omega_{X}^{\mu}(l) Q
$$

is the $\beta$ coupling while $H_{l}$ is the Hamiltonian for the lepton field alone. In (2) as in all the subsequent formalism, our notation is that the operator product $\omega \omega^{\prime} \omega^{\prime \prime} \cdots$ means $\sum_{k} \omega_{k} \omega_{k}{ }^{\prime} \omega_{k}{ }^{\prime \prime} \cdots$, where $k$ is a particle label. The only exception to this rule is the minor one that wherever $V$, the nuclear force operator, occurs a double summation is implied. Thus, in explicit form,

$$
V=\sum_{i j}{ }^{\prime} V_{i j}=\sum_{i j}{ }^{\prime} V_{i} V_{j}
$$

where the prime means that $i=j$ is excluded. Then, in the following discussion, where commutators with $V$ appear, we recognize that

$$
\begin{aligned}
(V, \omega) & =\sum_{i j}{ }^{\prime}\left(V_{i j}, \omega_{i}+\omega_{j}\right) \\
& =\sum_{i j}^{\prime}\left[\left(V_{i}, \omega_{i}\right) V_{j}+V_{i}\left(V_{j}, \omega_{j}\right)\right],
\end{aligned}
$$

where $(A, B) \equiv A B-B A$ and again $\omega=\sum_{k} \omega_{k}$. But

$$
\left(\omega, \omega^{\prime}\right)=\sum_{k}\left(\omega_{k}, \omega_{k}^{\prime}\right) .
$$

Our units are such that $c=\hbar=1$ and later, when the $\beta$-particle wave functions are introduced, $m=1$. In Eq. (2), $M_{n}$ and $M_{p}$ are the neutron and proton masses, respectively.

In (3) only electron emission is represented explicitly. The positron emission is obtained of course by adding the hermitian conjugate of $H_{\beta}$. In the following we consider electron emission wherever it is necessary to be explicit. For positron emission the results are obtained as usual by changing $Z$ to $-Z$ (and introducing the sign changes in the Fierz interference terms ${ }^{10}$ ) or, if the central field in which the $\beta$ particle moves is not a Coulomb field but is $V_{c}$, one changes $V_{c}$ to $-V_{c}$. The operators $\Omega_{X}{ }^{\mu}(N) \Omega_{X}{ }^{\mu}(l)$ in (3) are the usual contracted tensors in nucleon $(N)$ and lepton $(l)$ spaces and for convenience only a single tensor index $(\mu)$ is written explicitly. Of course, $X=S, V, T, A$, and $P$. If $\Omega_{X}(N)=\omega_{\mu}{ }^{N}$, then $\Omega_{X}{ }^{\mu}(l)=\psi^{*} \omega_{\mu} \zeta \varphi=L\left(\omega_{\mu}\right)$, where $\omega_{\mu}$ is

${ }^{9}$ L. L. Foldy and S. A. Wouthuysen, Phys. Rev. 78, 29 (1950), referred to as FW.

${ }_{10}$ M. Fierz, Z. Physik 104, 553 (1937). 
a Dirac operator in lepton space, $\varphi$ is a positive energy neutrino wave function, and $C\left(=i \beta \alpha_{2} K_{0}\right.$ with $K_{0}$ meaning complex conjugation) is the charge conjugation operator. The lepton covariant, referred to above, is just $L\left(\omega_{\mu}\right) . Q$ is the operator which changes neutron into proton. Except in special circumstances we need not make this operator explicit. We adopt the special representation

$$
\alpha=\left(\begin{array}{ll}
0 & \boldsymbol{\sigma} \\
\boldsymbol{\sigma} & 0
\end{array}\right), \quad \beta=\left(\begin{array}{cc}
1 & 0 \\
0 & -1
\end{array}\right)
$$

in the Dirac space notation. Then our sign convention is the one usually used in $\beta$-decay theory.

The FW transformation is applied to the total Hamiltonian $H$ with the purpose of removing odd operators in $H_{N}$ and $H_{\beta}$ to a given order. ${ }^{9}$ That is, the new Hamiltonian is

$$
H^{\prime}=e^{S} H e^{-S}=H+(S, H)+\frac{1}{2}(S,(S, H))+\cdots,
$$

and we restrict our attention to just the three terms written. However, as is well known, consistent results are obtained if in the double commutator only the mass term $(\sim-\beta M)$ is taken. To this order, the mass difference $M_{n}-M_{p}$ is not essential for the new $\beta$-decay interaction $\left(H_{\beta}{ }^{\prime}\right)$ but it will be for some subsequent results (see Sec. III). Also, to this order the transformation of $H_{l}$ does not contribute anything to $H_{\beta}{ }^{\prime}$. Superficially, it would appear that the expansion parameter is of order $p / M \sim v / c$ ( $v$ the nucleon velocity) but a more thorough investigation shows that, because of the parity properties of the $\mathbf{p}$ operator, the expansion parameter here is effectively $p^{2} / M^{2} \sim 10^{-2}$.

Writing

$$
H_{N}=H_{N}(e)+O_{1}, \quad H_{\beta}=H_{\beta}(e)+O_{2},
$$

where $H_{N}(e), H_{\beta}(e)$ are the respective even parts and $O_{1}, O_{2}$ the respective odd parts in Dirac space we have

$$
S=-\frac{\beta}{2 M}\left(O_{1}+O_{2}\right) \text {. }
$$

The commutator $(S,-\beta M)=-\left(O_{1}+O_{2}\right)$, so that these odd terms are removed by the canonical transformation. The commutator of $S$ and an even operator is odd and can be removed by another canonical transformation of the type (9). The even terms thus generated are of order $1 / M^{2}$ and are neglected.

For the nuclear force operator $V$ it is legitimate to assume a sum over pairs of terms which are symmetrical between the interacting nucleons. Hence there should be two types of interaction operators: direct products of even operators and of odd operators. The direct product of two even operators, $V_{e}$, gives rise to no difficulty; see Eq. (11) below. The prescription (9) for removing odd operators does not apply directly in the case of two-particle operators $\left(V_{0}\right)$. However, the removal of such terms can be effected ${ }^{11}$ and the resulting transformed operators appear only in terms of order $1 / M^{2}$. Consequently, these terms need not concern us further.

Applying successive transformations of the type (9) until all odd operators to the prescribed order disappear, we find

with

$$
H^{\prime}=H_{N}{ }^{\prime}+H_{\beta}{ }^{\prime}+H_{l}
$$

$$
H_{N}^{\prime}=-\frac{\beta p^{2}}{2 M}-\beta M-\frac{1}{2} \Delta \beta \tau_{z}+V_{e},
$$

where $\Delta=M_{n}-M_{p}$ and

$$
H_{\beta}{ }^{\prime}=-\frac{1}{2 M} \beta\left(O_{1}, O_{2}\right)_{+}+H_{\beta}(e)
$$

where $\left(O_{1}, O_{2}\right)_{+}=O_{1} O_{2}+O_{2} O_{1}$. Of course, terms quadratic in the coupling constants $g_{X}$ are dropped. The results given in (11) and (12) are identical with those obtained by the method of elimination of small components when carried out to the same order in $1 / M$.

With the representation $\left(2^{\prime}\right)$ the positive energy solutions correspond to $\beta=-1$. We set $\beta=-1$ (in the nuclear space) from this point on, realizing that no approximation is involved other than that involved in terminating the development given by (7). The zero order nuclear wave functions are now truly nonrelativistic ones. From (12) it is apparent that the $\beta$ interaction contains only even operators and that $H_{\beta}(e)$ which accounts for allowed transitions as well as unique matrix element transitions, is unaffected.

At this point one can begin to discern the role of the lepton covariant. The $S$ operator involves $\beta \boldsymbol{\alpha} \cdot \mathbf{p} / 2 M$ and a gradient operation on $L$ appears. In the pseudoscalar case this is $a b l$ that appears. This fact becomes apparent below when we explicitly compute the anticommutator of (12). However, it is enlightening to see how this happens when one uses the method of elimination of small components. For this purpose we use a simplified one-particle model, but this simplification does not change the essential ideas. Then, with the nuclear wave function in the form

$$
\Psi=\left(\begin{array}{l}
v \\
u
\end{array}\right)
$$

where $u, v$ are the large and small components, respec-

${ }^{11}$ In the case of a two-particle Hamiltonian it has been shown by Z. V. Chraplyvy, Phys. Rev. 91, 388 (1953), that a canonical transformation which removes $V_{0}$ can be constructed. While the generating function $S$ given in this reference is singular in the case of two particles of equal mass, this difficulty disappears if the canonical transformation is required to separate only states in which both particles have the same sign of the energy. Extension to the case of many particles presents no difficulty. [See Z. V. Chraplyvy, Phys. Rev. 92, 1310 (1953).] We wish to express our thanks to Dr. Chraplyvy for communicating these results before publication. 
tively, we have

$$
\int \beta \gamma_{5} L \equiv \int \Psi_{f}^{*} \beta \gamma_{5} L \Psi_{i}=-\int\left(v_{f}^{*} L u_{i}-u_{f}^{*} L v_{i}\right),
$$

where $L=L\left(\beta \gamma_{5}\right)$. Eliminating $v$ by

$$
\begin{aligned}
v & =-(W+M-V)^{-1} \mathbf{\sigma} \cdot \mathbf{p} u \\
& \approx-\frac{1}{2 M} \boldsymbol{\sigma} \cdot \mathbf{p} u
\end{aligned}
$$

since $W-M-V \ll 2 M$, we have

$$
\int \beta \gamma_{5} L=\frac{1}{2 M} \int u_{f}^{*}(\boldsymbol{\sigma} \cdot \mathbf{p} L) u_{i}
$$

where $\mathbf{p}$ acts only on $L$. The way in which the momentum of the nucleon, entering through the small component, introduces the lepton parameters (energy and $V_{c}$ ) is now clear.

For simplicity, we consider plane waves

$$
L=L_{P} e^{-i \mathbf{P} \cdot \mathbf{r}}
$$

where $L_{P}$ contains the lepton spinor amplitudes, and ${ }^{12}$

$$
\mathbf{P}=\mathbf{p}_{e}+\mathbf{q},
$$

with $\mathbf{p}_{e}$, $\mathbf{q}$ the electron and neutrino momenta, respectively. Then, with a retardation expansion,

$$
\int \beta \gamma_{5} L=-\frac{1}{2 M} \int \boldsymbol{\sigma} \cdot \mathbf{P} L_{P}(1-i \mathbf{P} \cdot \mathbf{r} \cdots) .
$$

The first term,

$$
-\frac{1}{2 M} L_{P} \mathbf{P} \cdot \int \boldsymbol{\sigma}
$$

gives rise to the second forbidden transitions and corresponds exactly to the contribution of $\int \beta \gamma_{5} \mathbf{r}$ in the $\mathrm{KU}$ representation. The second term gives rise to two terms:

and

$$
\frac{i}{4 M} \sum_{i j} B_{i j} P_{i} P_{j}
$$

$$
\frac{i}{6 M} P^{2} \int \boldsymbol{\sigma} \cdot \mathbf{r}
$$

The correction factor associated with the first is the same as that associated with $R_{i j}{ }^{\beta \gamma 5}$ in the KU representation. The second part has a correction factor which is the same as that associated with the matrix element of $\beta \gamma_{5} r^{2}$. In fact, we would say that

$$
i \int \boldsymbol{\sigma} \cdot \mathbf{r} \leftrightarrow-\int \beta \gamma_{5} r^{2},
$$

${ }^{12}$ In order to avoid confusion with the $\mathbf{p}$ operator we attach a subscript to the electron momentum. Later, where no confusion will arise we drop this subscript. in the sense that the matrix element on the left replaces that on the right with the same correction factor associated with each. In the $\mathrm{KU}$ theory the $\int \beta \gamma_{5} r^{2}$ term is supposed to be only a small correction to the $\int \beta \gamma_{5}$ contribution. It would there be called a third forbidden correction. However, there is no term corresponding to $\int \beta \gamma_{5}$, which is the matrix element appearing in the $\mathrm{RaE}$ analysis. Of course, we are using plane waves but the results shown below verify that this is no real restriction. The results obtained with this simplified model are fully substantiated by the application of the less restrictive FW transformation.

The question now arises as to whether the $0 \rightarrow 0$, yes, transition should be called third or first forbidden. We postpone the discussion of this until Sec. IV where the pseudoscalar correction factor is obtained. For leptons in a central field we shall show that the situation is very different and that for a Coulomb field, for example, one gets a correction factor whose largest contribution $(Z \neq 0)$ indeed vanishes in the $Z=0$ limit and it has been verified that the results reduce (for $Z=0$ ) to the correction factor implied by the above. The matrix element for the zero-rank tensor for nonfree leptons is again $\boldsymbol{S} \boldsymbol{\sigma} \cdot \mathbf{r}$. We expect to find the correction factor for this term, which makes the sole contribution to $0 \rightarrow 0$, yes, in the pseudoscalar case, to be strongly $Z$ - and energy-dependent.

\section{THE BETA-DECAY OPERATORS IN NONRELATIVISTIC FORM}

The recasting of the operators for forbidden transitions resulting from Eq. (12) may now be exhibited explicitly. The transformed (formerly odd) part of the interaction now consists of two types of terms: $\mathcal{E}$ operators in which there appears a p operator acting on the nuclear wave function and $\mathcal{E}^{\prime}$ operators in which p acts only on the lepton covariant. That is, we write

\begin{tabular}{|c|c|c|c|}
\hline $\begin{array}{l}\text { Inter- } \\
\text { action }\end{array}$ & {$\left[\Omega X^{\mu}(N) \Omega x^{\mu}(l)\right]_{\text {odd }}$} & $\mathcal{E} X$ & $\varepsilon x^{\prime}$ \\
\hline$V$ & $-L(\boldsymbol{\alpha}) \cdot \boldsymbol{\alpha}$ & $2 L(\boldsymbol{\alpha}) \cdot \mathbf{p}$ & $\begin{aligned} \mathbf{p} \cdot & L(\boldsymbol{\alpha}) \\
& +i \boldsymbol{\sigma} \cdot \mathbf{p} \times L(\boldsymbol{\alpha})\end{aligned}$ \\
\hline$T$ & $L(\boldsymbol{\beta} \boldsymbol{\alpha}) \cdot \boldsymbol{\beta} \boldsymbol{\alpha}$ & $-2 i L(\beta \boldsymbol{\alpha}) \cdot(\boldsymbol{\sigma} \times \mathbf{p})$ & $\begin{array}{l}-\mathbf{p} \cdot L(\boldsymbol{\beta} \boldsymbol{\alpha}) \\
\quad-i \boldsymbol{\sigma} \cdot \mathbf{p} \times L(\beta \boldsymbol{\alpha})\end{array}$ \\
\hline $\begin{array}{l}A \\
P\end{array}$ & $\begin{array}{l}-L\left(\gamma_{5}\right) \gamma_{5} \\
L\left(\beta \gamma_{5}\right) \beta \gamma_{5}\end{array}$ & $-\underset{\mathbf{0}}{-2 L\left(\gamma_{5}\right) \boldsymbol{\sigma} \cdot \mathbf{p}}$ & $\begin{array}{l}-\boldsymbol{\sigma} \cdot \mathbf{p} L\left(\gamma_{5}\right) \\
-\boldsymbol{\sigma} \cdot \mathbf{p} L\left(\gamma_{5}\right)\end{array}$ \\
\hline
\end{tabular}
(12) in the form

$$
H_{\beta}{ }^{\prime}=H_{\beta}(e)+\frac{1}{2 M} \sum_{X} g_{X}\left(\mathcal{E}_{X}+\mathcal{E}_{X}^{\prime}\right) Q,
$$

and

$H_{\beta}(e)=\left[-g_{S} L(\beta)+g_{V} L(1)-g_{T} \boldsymbol{\sigma} \cdot L(\beta \boldsymbol{\sigma})+g_{A} \boldsymbol{\sigma} \cdot L(\boldsymbol{\sigma})\right] Q$.

In Table I we list the odd part of $\Omega_{X}{ }^{\mu}(N) \Omega_{X}{ }^{\mu}(l)$ before and after the canonical transformation. Of course, we we may set $\beta=-1$ only for $\mathcal{E}$ and $\mathcal{E}^{\prime}$.

TABLE I. Beta-interaction operators in odd and even form. 
The vanishing of $\mathscr{E}_{P}$ confirms the results of the elementary considerations given above. In a formal manner of expression this result arises whenever one deals with a nonvector operator of the form of a direct product of a Pauli operator and the Dirac operator $\rho_{2}$. The only operator of this form is $\beta \gamma_{5}$. It is also obvious that, if $L\left(\beta \gamma_{5}\right)$ were treated as a constant, the pseudoscalar contribution disappears. The fact that the only nonvanishing term contains $\boldsymbol{\sigma} \cdot \mathbf{p} L\left(\beta \gamma_{5}\right)$ and not $L\left(\beta \gamma_{5}\right)$ implies that the correction factors may very well be changed in a serious manner, as turns out to be the case.

We shall show in succeeding paragraphs that, except for the pseudoscalar interaction, only the (originally) even operators $H_{\beta}(e)$ and the $\mathcal{E}$ operators are important. Thus, leaving aside the $\mathcal{E}^{\prime}$ operators for the moment, it is clear that for the $S, V, T, A$ interactions the correction factors are identical with the well-known results. ${ }^{1}$ The only change, as is evident from Table I, is a replacement of every odd operator by an even one. Thus, we make the replacements $:^{13}$

$$
\begin{gathered}
\boldsymbol{\alpha} \rightarrow-(1 / M) \mathbf{p}, \quad i \beta \boldsymbol{\alpha} \rightarrow(1 / M) \boldsymbol{\sigma} \times \mathbf{p}, \\
\gamma_{5} \rightarrow(1 / M) \boldsymbol{\sigma} \cdot \mathbf{p} .
\end{gathered}
$$

This correspondence between operators, and of course between matrix elements, extends to all higher rank tensors formed by adjoining the position vector $\mathbf{r}$. In so doing $\mathbf{p}$ must operate to the right of $\mathbf{r}$. Thus [see Eq. (26d) below],

$$
A_{i j} \rightarrow-\frac{1}{M} \tau_{i j}(\mathbf{r}, \mathbf{p}) ; A_{i j} \stackrel{i}{\rightarrow} \underset{M}{M} \tau_{i j}(\mathbf{r}, \boldsymbol{\sigma} \times \mathbf{p}),
$$

et simile. Here $\tau_{i j}(\mathbf{a}, \mathbf{b})$ is the matrix element of the $i j$ component of a symmetrical second rank tensor with vanishing trace.

$$
\tau_{i j}(\mathbf{a}, \mathbf{b})=\int\left(a_{i} b_{j}+a_{j} b_{i}-\frac{2}{3} \mathbf{a} \cdot \mathbf{b} \delta_{i j}\right) .
$$

Particular attention must be given to the order of the operators because of the noncommutation of $\mathbf{r}$ and $\mathbf{p}$.

One may now raise the question as to whether the $\mathscr{E}_{X}{ }^{\prime}$ operators effect an appreciable change in the correction factors for interactions other than $P$. The first answer which one is tempted to give is that, since $\mathcal{E}^{\prime}$ involves the lepton momenta while $\mathcal{E}$ involves the nucleon momentum, the former should make very small contributions in comparison with the latter. Actually, this reasoning is not entirely correct although, for all practical purposes, the conclusion is correct. In order to investigate this question it suffices to treat the leptons as free particles. Then a term-by-term comparison of the matrix elements, together with correction factors,

\footnotetext{
${ }^{13}$ Essentially the same results were given by $T$. Ahrens and E. Feenberg, Phys. Rev. 86, 64 (1951). We do not check their sign in the case of $\gamma_{5}$ and, moreover, we feel that the present procedure places the operator correspondence on a firmer foundation where whatever approximation is involved may be explicitly brought to light
}

for the conventional and transformed operators, may be made. For example, for the tensor interaction, the customary matrix element would be written, with $L(\beta \boldsymbol{\alpha})=\mathbf{L}_{T} e^{-i \mathbf{P} \cdot \mathbf{r}}$,

$$
\begin{aligned}
M_{T}(K U)= & \int \beta \boldsymbol{\alpha} \cdot \mathbf{L}_{T}(1-i \mathbf{P} \cdot \mathbf{r} \cdots) \\
= & \mathbf{L}_{T} \cdot \int \beta \boldsymbol{\alpha}-\frac{i}{2} \sum_{i j} P_{i} L_{T j} \\
& \times\left[A_{i j}{ }^{\beta}+\int \beta(\boldsymbol{\alpha} \times \mathbf{r})_{i j}+\frac{2}{3} \int \beta(\boldsymbol{\alpha} \cdot \mathbf{r}) \delta_{i j}\right] \\
= & \mathbf{L}_{T} \cdot \int \beta \boldsymbol{\alpha}-\frac{i}{3} \mathbf{P} \cdot \mathbf{L}_{T} \int \beta \boldsymbol{\alpha} \cdot \mathbf{r}+\frac{i}{2}\left(\mathbf{P} \times \mathbf{L}_{T}\right) \\
& \quad \int \beta \boldsymbol{\alpha} \times \mathbf{r}-\frac{i}{2} \sum_{i j} P_{i} L_{T j} A_{i j}{ }^{\beta} .
\end{aligned}
$$

For the matrix element of the transformed operators one finds

$$
\begin{gathered}
M_{T}=\frac{1}{2 M} \int(1-i \mathbf{P} \cdot \mathbf{r})\left[\mathbf{P} \cdot \mathbf{L}_{T}+i \boldsymbol{\sigma} \cdot \mathbf{P} \times \mathbf{L}_{T}-2 i \mathbf{L}_{T} \cdot \mathbf{\sigma} \times \mathbf{p}\right] \\
=\frac{1}{2 M}\left\{\mathbf{P} \cdot \mathbf{L}_{T} \int 1+i \mathbf{P} \times \mathbf{L}_{T} \cdot \int \boldsymbol{\sigma}-2 i \mathbf{L}_{T} \cdot \int \boldsymbol{\sigma} \times \mathbf{p}\right. \\
\quad+\sum_{i j} L_{T i} P_{i} \tau_{i j}(\mathbf{r}, \sigma \times \mathbf{p})+\mathbf{P} \times \mathbf{L}_{T} \\
\left.\cdot \int \mathbf{r} \times(\mathbf{\sigma} \times \mathbf{p})-\frac{2}{3} \mathbf{L}_{T} \cdot \mathbf{P} \int \mathbf{\sigma} \cdot \mathbf{r} \times \mathbf{p}\right\} \cdot
\end{gathered}
$$

The comparison shows that the correspondence between operators is

$$
\begin{aligned}
& i \int \beta \boldsymbol{\alpha} \rightarrow \frac{1}{M} \int \boldsymbol{\sigma} \times \mathbf{p}, \\
& i \int \beta \boldsymbol{\alpha} \cdot \mathbf{r} \rightarrow-\frac{3}{2 M} \int\left(1-\frac{2}{3} \boldsymbol{\sigma} \cdot \mathbf{r} \times \mathbf{p}\right), \\
& i \int \beta \boldsymbol{\alpha} \times \mathbf{r} \rightarrow \frac{1}{M} \int[i \boldsymbol{\sigma}+\mathbf{r} \times(\boldsymbol{\sigma} \times \mathbf{p})], \\
&-i A_{i j}{ }^{\beta} \rightarrow \frac{1}{M} \tau_{i j}(\mathbf{r}, \boldsymbol{\sigma} \times \mathbf{p}) .
\end{aligned}
$$

It is seen that in the second and third of these $(26 \mathrm{~b}$ and 26c) both $\mathcal{E}$ and $\mathcal{E}^{\prime}$ operators contribute. The reason is almost trivial from a consideration of parity. The $\mathcal{E}$ operators involving $\mathbf{p}$ must combine with the $\mathbf{P} \cdot \mathbf{r}$ term of the retardation expansion while the $\mathcal{E}^{\prime}$ operators combine with the first term. Note that both $\beta \boldsymbol{\alpha} \cdot \mathbf{r}$ and $\beta \boldsymbol{\alpha} \times \mathbf{r}$ correspond to second-order forbidden. For $\beta \boldsymbol{\alpha}$, 
TABLE II. Transformation of $\varepsilon$ operators, see Eq. (29).

\begin{tabular}{cccc}
\hline \hline Interaction & $n$ & $x$ & $\chi^{\prime}$ \\
\hline$V$ & 1 & $\mathbf{p}$ & $\mathbf{r}$ \\
$T$ & 2 & $\mathcal{T}_{i j}(\mathbf{r}, \mathbf{p})$ & $\frac{1}{2} \tau_{i j}(\mathbf{r}, \mathbf{r})=R_{i j}$ \\
$A$ & 1 & $\mathbf{\sigma} \times \mathbf{p}$ & $\mathbf{\sigma} \times \mathbf{r}$ \\
\hline \hline
\end{tabular}

which enters in first-order forbidden, the $\mathscr{E}$ operator can combine with the large leading term of the exponential and the $\mathcal{E}^{\prime}$ operator makes an extremely trivial correction. For the case of the second rank tensor $\tau_{i j}(\mathbf{r}, \boldsymbol{\sigma} \times \mathbf{p})$ which is, of course, a second forbidden matrix element, the $\mathcal{E}^{\prime}$ operator does not contribute a like term until one includes $(\mathbf{P} \cdot \mathbf{r})^{2}$ terms from the exponential which, again, would constitute a very small correction. We therefore conclude that for first forbidden transitions the $\mathcal{E}^{\prime}$ operators are unimportant while for second forbidden transitions they are unimportant for $|\Delta J|=2$; but for tensors of zero and first rank (maximum $\Delta J=0,1$ ) they must be considered and both $\mathcal{E}$ and $\mathcal{E}^{\prime}$ enter. That they make contributions of comparable magnitude is obvious from the fact that for low-lying $\beta$-emitting states $p \rho \sim 1, \rho$ being the nuclear radius. Also, both $\mathcal{E}$ and $\mathcal{E}^{\prime}$ operators are necessary in order that the canonical transformation preserve the hermitian character of the operators.

The same conclusion follows with the vector and axial vector interactions. The $\mathcal{E}$ and $\mathcal{E}^{\prime}$ operators are equally involved for second forbidden transitions with tensors of rank 0 or 1 . Thus, for the $V$ interaction,

$$
\int \boldsymbol{\alpha} \cdot \mathbf{r} \rightarrow \frac{3 i}{2 M} \int\left(1+\frac{2}{3} i \mathbf{r} \cdot \mathbf{p}\right), \quad \int \boldsymbol{\alpha} \times \mathbf{r} \rightarrow \frac{1}{M} \int(\boldsymbol{\sigma}+\mathbf{r} \times \mathbf{p})
$$

and for the $A$ interaction

$$
\int \gamma_{5} \mathbf{r} \rightarrow-\frac{i}{2 M} \int[\boldsymbol{\sigma}+2 i \mathbf{r}(\boldsymbol{\sigma} \cdot \mathbf{p})] .
$$

These conclusions are not changed when one removes the restriction of free leptons and considers the fact that the electron moves in some central field. Moreover, one can easily extend the rule to the effect that for all orders of forbiddenness $n$ equal to or higher than second, the correction factors would be appreciably modified for $(\Delta J)_{\max }<n$. However, if one accepts the present experimental evidence that the beta interaction contains both Fermi and Gamow-Teller constitutents and that both types are present in appreciable amount, then all these types of transitions with a modified correction factor become academic. They represent small contributions to a lower order of forbiddenness where the $\mathcal{E}^{\prime}$ operator makes a negligible contribution and the correction factor is, to a very high degree of accuracy, just that given in the usual theory. ${ }^{1,14}$

\footnotetext{
${ }^{14}$ See also E. Greuling, Phys. Rev. 61, 568 (1942). Also D. L. Pursey, Phil. Mag. 42, 1193 (1951); A. M. Smith, Phys. Rev. 82, 955 (1951).
}

It will be recognized that the transformation of the operators carried out above does not depend on any detailed knowledge of the nuclear forces. ${ }^{11}$ It is possible to simplify some of the $\mathcal{E}$ operators introduced above but this simplification does depend on more specific assumptions concerning these forces. Thus, for any operator $\chi^{\prime}$ one may write

$$
\left(W_{f}-W_{i}\right) \int \chi^{\prime}=\int\left(H, \chi^{\prime}\right)
$$

where $W_{f}$ and $W_{i}$ are the final and initial eigenvalues of the nuclear Hamiltonian $H=H_{N}{ }^{\prime}$, see Eq. (11). One now attempts to find an operator $\chi^{\prime}$ such that $\left(p^{2}, \chi^{\prime}\right)=-2 i \chi$ for a given $\chi$. The term $\frac{1}{2} \Delta \tau_{z}$ in (11) contributes to $\left(H_{N^{\prime}}, \chi^{\prime}\right)$ and now the charge transformation operator $Q$ must be placed in evidence.

$$
\frac{1}{2} \Delta\left(\tau_{z}, \chi^{\prime} Q\right)=\frac{1}{2} \Delta \chi^{\prime}\left(\tau_{z}, Q\right)=-\Delta \chi^{\prime} Q,
$$

since $\chi^{\prime}$ is charge independent. Then, it follows that

$$
\int \chi=i M\left\{\left(\Delta-W_{0}\right) \int \chi^{\prime}-\int\left(V, \chi^{\prime}\right)\right\},
$$

where $W_{0}=W_{i}-W_{f}$ is the $\beta$-energy release.

The $\chi$ operators ( $\mathscr{E}$ type) to be considered are $\mathbf{p}$, $\tau_{i j}(\mathbf{r}, \mathbf{p})$ from the $V$ interaction; $\boldsymbol{\sigma} \times \mathbf{p}, \tau_{i j}(\mathbf{r}, \boldsymbol{\sigma} \times \mathbf{p})$ from the $T$ interaction and $\boldsymbol{\sigma} \cdot \mathbf{p}$ from the $A$ interaction. We limit our consideration to first and second forbidden transitions. Then with the aid of

$$
\left(\mathbf{p}^{2}, \mathbf{r}\right)=-2 i \mathbf{p}, \quad\left(p^{2}, r_{j} r_{k}\right)=-2 \delta_{j k}-2 i\left(r_{j} p_{k}+r_{k} p_{j}\right),
$$

one can find the appropriate $\chi^{\prime}$ operator for all these tensors with the exception of $\tau_{i j}(\mathbf{r}, \boldsymbol{\sigma} \times \mathbf{p})$. The results are exhibited in Table II. For the tensor $\tau_{i j}(\mathbf{r}, \boldsymbol{\sigma} \times \mathbf{p})$ one would expect that the $\chi^{\prime}$ operator is $\tau_{i j}(\mathbf{r}, \boldsymbol{\sigma} \times \mathbf{r})$ $=T_{i j}$, in the usual notation. ${ }^{1}$ Actually,

$$
\left(p^{2}, \tau_{i j}(\mathbf{r}, \boldsymbol{\sigma} \times \mathbf{r})\right)=-4 i \tau_{i j}(\mathbf{r}, \boldsymbol{\sigma} \times \mathbf{p})+2 i \tau_{i j}(\boldsymbol{\sigma}, \mathbf{L}),
$$

where $\mathbf{L}=\mathbf{r} \times \mathbf{p}$ is the orbital angular momentum operator.

If one assumes that the nuclear force operator is velocity-independent, which is plausible as a first approximation, ${ }^{15}$ the $\boldsymbol{\alpha} \rightarrow-\mathbf{p} / M$ operator is replaced by $-i\left(\Delta-W_{0}\right) \mathbf{r}$. Thus, the $\Lambda$ introduced by Feenberg ${ }^{13}$ is given by ${ }^{16}$

$$
\Lambda=\frac{-2 i \rho}{\alpha Z} \frac{\int \alpha}{\int \mathbf{r}}=\frac{2 \rho}{\alpha Z}\left(W_{0}-\Delta\right) \approx \frac{A^{\frac{1}{3}}}{Z}\left(W_{0}-\Delta\right) .
$$

\footnotetext{
${ }^{15}$ Strictly speaking, the commutator $(V, \mathbf{r})$ does not vanish unless one can also neglect the nuclear Coulomb energy or the charge dependent interactions. Here we recall the suppressed $Q$ operator, see Ahrens and Feenberg (reference 13). The comments based on the assumption that $(V, \mathbf{r})=0$ should therefore be applicable to light nuclei.

${ }_{16}$ See also D, L. Pursey, reference 14.
} 
The same assumption of velocity independence of the forces permits the elimination of $\tau_{i j}(\mathbf{r}, \mathbf{p})$ which is replaced by $i M R_{i j}$ which also appears from the even part $H_{\beta}(e)$ of the interaction. The number of operators involved in forbidden transitions is thus somewhat reduced and there is less arbitrariness in fitting $\beta$ spectra than was hitherto supposed. If one considers a mixture of two interactions, the ratio of matrix elements multiplied with coupling constants is, in some cases, reduced to simply the ratio of coupling constants. In this way, one may obtain vital information concerning the composition of the $\beta$ interaction from the shapes of some forbidden spectra.

It may be seen that for zero spin change, parity change yes, transitions, the only operator involved in both $P$ and $T$ interactions is $\boldsymbol{\sigma} \cdot \mathbf{r}$. This will appear explicitly in Sec. IV. Thus, the RaE spectrum provides the possibility of fixing $g_{P} / g_{T}$ if the transition corresponds to $\Delta J=0$. Here no assumption about nuclear forces is involved. For the $A$ interaction there are two zero rank, odd parity tensors : $\boldsymbol{\sigma} \cdot \mathbf{r}$ and $\boldsymbol{\sigma} \cdot \mathbf{p}$. Only if one assumes that $(V, \boldsymbol{\sigma} \cdot \mathbf{r})=0$ (spin-dependent nuclear forces are sufficient but may not be necessary) could one reduce these two operators to a single one.

\section{THE CORRECTION FACTORS}

The calculation of the correction factors, which are presumably pertinent for $\mathrm{RaE}$, is a fairly simple one. However, we do not wish to restrict our consideration to this case and prefer to consider a more general situation in which one need not specify the parity or spin change (tensor rank) at the start. Instead, the general correction factor for the $P$, or any other, interaction can be given with no more effort than is involved in calculating special cases. The formalism which is ideally suited for our purpose is that which is involved in a complete utilization of the angular momentum representation. We have found this procedure to be highly useful not only for the calculation of correction factors but also for the determination of nuclear matrix elements. The formalism is therefore presented in the following in some detail.

We designate the quantum numbers for the electron and neutrino by $\kappa, \mu$, and $\kappa_{\nu}, \mu_{\nu}$, respectively. Here $\kappa$, a nonzero integer, gives both the angular momentum $j$ according to

$$
j=|\kappa|-\frac{1}{2}
$$

and the parity $(-)^{l \kappa+1}$ according to

$$
l_{\kappa}=|\kappa|+\frac{1}{2}\left(S_{\kappa}-1\right),
$$

where $S_{\kappa}$ is the sign of $\kappa$. Thus, $j=l_{\kappa}-\frac{1}{2} S_{\kappa}$ and $\left|b_{\kappa}-b_{-\kappa}\right|$ $=1$. The eigenvalue of $j_{z}$ is $\mu$, the magnetic quantum number.

In terms of this notation the differential energy spectrum is

$$
N(W)=\frac{2}{\pi} \frac{W}{p} \sum_{\kappa \kappa_{\nu}} \sum_{\mu \mu_{\nu}}\left|\int H_{\beta}^{\prime}\right|^{2},
$$

and the correction factor is

$$
C_{\beta}=\frac{4 \pi^{2}}{p^{2} q^{2} F_{0}} \sum_{\kappa \kappa \nu} \sum_{\mu \mu_{\nu}}\left|\int H_{\beta}^{\prime}\right|^{2}
$$

Here $F_{0}$ is the well-known Fermi function. ${ }^{14}$

In an arbitrary central field the electron wave functions are

$$
\psi=\left(\begin{array}{c}
-i f_{\kappa} \chi_{-\kappa^{\mu}} \\
g_{\kappa} \chi_{\kappa}{ }^{\mu}
\end{array}\right)
$$

and a similar representation of the neutrino wave function $\varphi$ with radial wave functions $F_{\kappa \nu}$ and $G_{\kappa_{\nu}}$ in place of $f_{\kappa}, g_{\kappa}$. With the usual sign convention $\left[H \psi=\left(-\boldsymbol{\alpha} \cdot \mathbf{p}-\beta+V_{c}\right) \psi\right]$, the radial wave equation, of which explicit use is made below, is

$$
\frac{d}{d r}\left(\begin{array}{l}
f \\
g
\end{array}\right)=\left(\begin{array}{cc}
(\kappa-1) / r & -\left(W-1-V_{c}\right) \\
W+1-V_{c} & -(\kappa+1) / r
\end{array}\right)\left(\begin{array}{l}
f \\
g
\end{array}\right),
$$

and for the neutrino we set the central field $V_{c}=0$ and replace $W \pm 1$ by $q$. The $f_{\kappa}$ and $g_{\kappa}$ are real and, for a Coulomb field are just $(\pi p / W)^{\frac{1}{2}}$ times those explicitly given elsewhere. ${ }^{17}$ For the neutrino,

$$
F_{\kappa}=S_{\kappa} q j_{l(-\kappa)}(q r), \quad G_{\kappa}=q j_{l(\kappa)}(q r),
$$

where $j_{l}$ is the spherical Bessel function. Throughout, the normalization corresponds to one particle in a sphere of unit radius.

In Eq. (36) the spin-angular functions are the usual Pauli spinors:

$$
\chi_{\kappa}{ }^{\mu}(\mathfrak{r})=\sum_{\tau} C\left(l_{\kappa} \frac{1}{2} j ; \mu-\tau, \tau\right) \chi_{\frac{1}{2}}{ }^{\tau} Y_{l \kappa}{ }^{\mu-\tau}(\mathfrak{r}),
$$

where the $C$ coefficients are the usual vector addition coefficients

$$
\left[C\left(j_{1} j_{2} j_{3} ; m_{1} m_{2}\right) \equiv\left(j_{1} j_{2} j_{3} m_{1}+m_{2} \mid j_{1} j_{2} m_{1} m_{2}\right)\right] .
$$

Their properties as well as those of the $\chi_{\kappa}{ }^{\mu}$ spinors are summarized elsewhere. ${ }^{18}$ In (39) $\mathfrak{r}$ is the unit vector. Also $\chi_{\frac{1}{2}}{ }^{\tau}$ is a spin eigenfunction $\left(\tau= \pm \frac{1}{2}\right)$.

In forming the pseudoscalar lepton covariant we use

$$
\beta \gamma_{5} C=i \sigma_{2} K_{0}=T,
$$

where $T$ is the time-reversal operator, and observe that

$$
T \chi_{\kappa}{ }^{\mu}=(-)^{l_{\kappa-j+\mu+1}} \chi_{\kappa}{ }^{-\mu} .
$$

The procedure for evaluating matrix elements of any operator (such as those of Table I) is simplified enormously if one avoids a retardation expansion. This expansion, which is simply carried out at the end of

17 M. E. Rose, Phys. Rev. 51, 484 (1937). Our present notation differs only in the explicit use of $\kappa$ as an index and also in that $Y_{l}{ }^{m}$ as here defined differs by a factor $(-)^{m}$ from the definition in Eq. (2) of this reference.

${ }^{18}$ G. Racah, Phys. Rev. 62, 438 (1942); L. C. Biedenharn and M. E. Rose, Revs. Modern Phys. 25, 729 (1953) and Rose, Biedenharn, and Arfken, Phys. Rev. 85, 5 (1952). 
the calculation, would, if performed at the beginning, necessitate a separate treatment of each term and the rather involved procedure whereby irreducible tensors are introduced. In our case these irreducible tensors appear automatically. For purposes of illustration the calculation of the pseudoscalar matrix element is displayed.

We use the notation

$$
\begin{aligned}
H_{P} & =\frac{1}{2 M} \mathbf{\sigma} \cdot \mathbf{p} L\left(\beta \gamma_{5}\right) \\
& =\frac{1}{2 M} \sum_{\xi}(-1)^{\xi} \sigma_{-\xi} p_{\xi} L\left(\beta \gamma_{5}\right),
\end{aligned}
$$

where $\xi= \pm 1,0$ and for any operator

$$
A_{ \pm 1}=\mp 2^{-\frac{1}{2}}\left(A_{x} \pm i A_{y}\right), \quad A_{0}=A_{z} .
$$

Then from (36), (40), and $\left(40^{\prime}\right)$,

$$
\begin{array}{r}
L\left(\beta \gamma_{5}\right)=\exp \left[i \pi\left(l_{\kappa_{\nu}}-j_{\nu}-\mu_{\nu}\right)\right]\left[g _ { \kappa } G _ { \kappa _ { \nu } } \left(\chi_{\kappa}{ }^{\mu}, \chi_{\kappa_{\nu}}{ }^{\left.-\mu_{\nu}\right)}\right.\right. \\
\left.-f_{\kappa} F_{\kappa_{\nu}}\left(\chi_{-\kappa}{ }^{\mu}, \chi-\kappa_{\nu}{ }^{-\mu_{\nu}}\right)\right] .
\end{array}
$$

The inner product of the spinors is readily evaluated from (39) and the coupling relation

$$
\begin{array}{r}
Y_{l^{m}}(\mathfrak{r}) Y_{l^{\prime}}{ }^{m^{\prime}}(\mathfrak{r})=\sum_{\lambda}\left[\frac{(2 l+1)\left(2 l^{\prime}+1\right)}{4 \pi(2 \lambda+1)}\right]^{\frac{1}{2}} C\left(l l^{\prime} \lambda, 00\right) \\
\quad \times C\left(l l^{\prime} \lambda ; m m^{\prime}\right) Y_{\lambda}{ }^{m+m^{\prime}}(\mathfrak{r}) .
\end{array}
$$

The vector addition coefficients imply that $l, l^{\prime}$, and $\lambda$ form a triangle. That is, $\left|l-l^{\prime}\right| \leqslant \lambda \leqslant l+l^{\prime}$, etc. In general, we refer to such a triangle relation by stating that $\Delta\left(l l^{\prime} \lambda\right)$ exists. Also, $C\left(l l^{\prime} \lambda ; 00\right)=0$ unless the parity rule $\left(l+l^{\prime}+\lambda=\right.$ even integer $)$ is satisfied.

The spin projection quantum number sum is readily carried out by using the orthonormal properties of the unitary $C$ coefficients after one performs a Racah ${ }^{18}$ recoupling according to the prescription:

$$
\begin{aligned}
C\left(j_{1} j_{2} j ;\right. & \left.m_{1} m_{2}\right) C\left(j j_{3} j_{4} ; m_{1}+m_{2}, m_{3}\right) \\
= & \sum_{s}[(2 s+1)(2 j+1)]^{\frac{1}{2}} C\left(j_{2} j_{3} s ; m_{2} m_{3}\right) \\
& \times C\left(j_{1} s j_{4} ; m_{1}, m_{2}+m_{3}\right) W\left(j_{1} j_{2} j_{4} j_{3} ; j s\right),
\end{aligned}
$$

where $W(a b c d ; e f)$ is a Racah coefficient, ${ }^{19}$ implying the existence of $\Delta(a b e), \Delta(c d e), \Delta(a c f), \Delta(b d f)$. The result

\footnotetext{
${ }^{19}$ The properties of these coefficients are discussed in Biedenharn, Blatt, and Rose, Revs. Modern Phys. 24, 249 (1952). See also G. Racah, reference 18 . Numerical values are given by L. C. Biedenharn, Oak Ridge National Laboratory Report ORNL-1098 (unpublished). These tables also give numerical values of $Z$ (abcd;ef) see J. M. Blatt and L. C. Biedenharn, Revs. Modern Phys. 24, 258 (1952) which is related to our $R$ coefficients, introduced in Eqs. (45) and (46), by

$$
R_{\lambda}\left(\kappa \kappa^{\prime}\right)=(-)^{l \kappa^{\prime}+j^{\prime}-\frac{1}{2}} i^{\lambda-l \kappa-l \kappa^{\prime}}(2 \lambda+1)^{-\frac{1}{2}} Z\left(l_{\kappa} j l_{\kappa^{\prime}} j^{\prime} ; \frac{1}{2} \lambda\right) .
$$

In all applications $\lambda-l-l_{\kappa^{\prime}}$ is an even integer so that both $R$ and $Z$ coefficients are real.
}

to be used in (42) is

$$
\begin{aligned}
\left(\chi_{\kappa}{ }^{\mu}, \chi_{\kappa^{\prime}}{ }^{\prime}\right)=(4 \pi)^{-\frac{1}{2}}(-)^{l_{\kappa}{ }^{\prime-} j^{\prime}+\mu+1} \sum_{\lambda} R_{\lambda}\left(\kappa \kappa^{\prime}\right) \\
\\
\quad \times C\left(j j^{\prime} \lambda ;-\mu, \mu^{\prime}\right) Y_{\lambda}^{\mu^{\prime}-\mu}(\mathfrak{r}),
\end{aligned}
$$$$
\text { where }^{19}
$$

$R_{\lambda}\left(\kappa \kappa^{\prime}\right)=(-)^{l_{\kappa}+j^{\prime}-\lambda-\frac{1}{2}}$

$$
\begin{array}{r}
\times\left[\frac{(2 j+1)\left(2 j^{\prime}+1\right)\left(2 l_{\kappa}+1\right)\left(2 l_{\kappa^{\prime}}+1\right)}{2 \lambda+1}\right]^{\frac{1}{2}} \\
\times C\left(l_{\kappa} l_{\kappa^{\prime}} \lambda ; 00\right) W\left(j j^{\prime} l_{\kappa^{\prime}} l_{\kappa^{\prime}} ; \lambda \frac{1}{2}\right) .
\end{array}
$$

To apply the $\boldsymbol{\sigma} \cdot \mathbf{p}$ operator to $L\left(\beta \gamma_{5}\right)$, we use

$$
\begin{aligned}
\nabla_{m} Y_{l}{ }^{M}(\mathfrak{r}) \Phi(r) \\
=\left(\frac{l+1}{2 l+3}\right)^{\frac{1}{2}} C(l 1 l+1 ; M m) Y_{l+1}{ }^{M+m} D_{-}(l) \Phi \\
\quad-\left(\frac{l}{2 l-1}\right)^{\frac{1}{2}} C(l 1 l-1 ; M m) Y_{l-1}{ }^{M+m} D_{+}(l) \Phi,
\end{aligned}
$$

where $\Phi$ is any radial function and

$$
D_{-}(l)=\frac{d}{d r}-\frac{l}{r}, \quad D_{+}(l)=\frac{d}{d r}+\frac{l+1}{r} .
$$

We are now in a position to introduce the irreducible tensors $T_{L l} l^{m}(\mathbf{A}, \mathbf{B})$ for any two vector operators $\mathbf{A}, \mathbf{B}$. These are defined by

$$
\begin{aligned}
T_{L l} l^{m}(\mathbf{A}, \mathbf{B})=\sum_{m^{\prime}} C(1 l L ;- & \left.m^{\prime}, m+m^{\prime}\right) \\
& \times \mathcal{Y}_{l^{m^{\prime}+m}}(\mathbf{A}) \mathcal{Y}_{1}{ }^{-m^{\prime}}(\mathbf{B}),
\end{aligned}
$$

and hence the inverse

$$
\mathcal{Y}_{l}{ }^{m}(\mathbf{A}) \mathcal{Y}_{1}{ }^{m^{\prime}}(\mathbf{B})=\sum_{L} C\left(1 l L ; m^{\prime} m\right) T_{L l^{m+m^{\prime}}}(\mathbf{A}, \mathbf{B})
$$

Here $\mathcal{Y}_{l} m(\mathbf{A})$ is a solid harmonic of degree $b$. Where $\mathbf{A}=\mathfrak{r}, \quad Y_{l}{ }^{m}=Y_{l}{ }^{m}$, of course. The rank of the tensor $T_{L l} l^{m}$ is $L$, corresponding to the fact that $T_{L l^{m}}$ transforms under 3-space rotations according to the $2 L+1$ dimensional representation of the rotation group..$^{20}$ The condition that $\Delta(L l 1)$ exists will be noted. The parity of $T_{L l} l^{m}(\mathbf{A B})$ is $\pi_{A}^{l} \pi_{B}$, so that if $\mathbf{A}=\mathfrak{r}, \mathbf{B}=\boldsymbol{\sigma}$ the parity is $(-)^{l}$ while for $\mathbf{A}=\mathfrak{r}, \mathbf{B}=\mathbf{p}$ or $\boldsymbol{\sigma} \times \mathbf{p}$ the parity is $(-)^{l+1}$.

Applying $\left(48^{\prime}\right)$ to $(47)$, one obtains for any B but with $\mathbf{A}=\mathfrak{r}$,

$$
\begin{aligned}
\mathbf{B} \cdot \mathbf{p} Y_{l^{M}} \Phi=i\left(\frac{4 \pi}{3}\right)^{\frac{1}{2}}\left\{\left(\frac{l+1}{2 l+1}\right)^{\frac{1}{2}} D_{-}(l) \Phi T_{l, l+1}{ }^{M}(\mathfrak{r}, \mathbf{B})\right. \\
\left.-\left(\frac{l}{2 l+1}\right)^{\frac{1}{2}} D_{+}(l) \Phi T_{l, l-1}{ }^{M}(\mathfrak{r}, \mathbf{B})\right\} .
\end{aligned}
$$

${ }^{20}$ E. P. Wigner, Gruppentheorie (Friedrich Vieweg und Sohn, Braunschweig, 1931). 
Then the operator $H_{P}$ becomes

$$
\begin{aligned}
H_{P}=\frac{(-)^{m} 3^{-\frac{1}{2}} i}{2 M} \sum_{\lambda} C\left(j j_{\nu} \lambda ;-\mu,-\mu_{\nu}\right)(2 \lambda+1)^{-\frac{1}{2}} \\
\quad \times\left[(\lambda+1)^{\frac{1}{2}} T_{\lambda \lambda+1}{ }^{-m}(\mathfrak{r}, \boldsymbol{\sigma}) D_{-}(\lambda)\right. \\
\left.-\lambda^{\frac{1}{2}} T_{\lambda \lambda-1}{ }^{-m}(\mathfrak{r}, \boldsymbol{\sigma}) D_{+}(\lambda)\right] \\
\quad \times\left[R_{\lambda}\left(-\kappa,-\kappa_{\nu}\right) f_{\kappa} F_{\kappa_{\nu}}-R_{\lambda}\left(\kappa, \kappa_{\nu}\right) g_{\kappa} G_{\kappa_{\nu}}\right],
\end{aligned}
$$

where $m=\mu+\mu_{\nu}$.

Each term in the $\lambda$ summation corresponds to the angular momentum selection rule that $\Delta\left(j j_{\nu} \lambda\right)$ exists and the parity selection rule $\pi=(-)^{\lambda+1}$, that is, $\pi_{i} \pi_{f}=\pi$, where $\pi_{i}$ and $\pi_{f}$ are initial and final state parities. The order of forbiddenness is, in this case, $n=\lambda+1$. On forming the square of the matrix element and summing over $\mu$ and $\mu_{\nu}$, it is seen that tensors of different rank cannot interfere, as expected. In fact, if we use the notation

and

$$
H_{P}=i \sum_{\lambda}\left(S_{\lambda}+T_{\lambda \lambda+1}{ }^{-m}+S_{\lambda}-T_{\lambda \lambda-1}{ }^{-m}\right)
$$

$$
I_{\lambda}(a, b)=\sum_{m} \int T_{\lambda \lambda+a}{ }^{-m}\left(\int T_{\lambda \lambda+b}{ }^{-m}\right)^{*}
$$

which is the (real) invariant combination of matrix elements, one obtains

$$
\begin{aligned}
\sum_{\mu \mu_{\nu}}\left|\int H_{P}\right|^{2}=\sum_{\lambda}\left[\left(S_{\lambda^{+}}\right)^{2} I_{\lambda}(11)\right. & +2 S_{\lambda}+S_{\lambda}^{-} I_{\lambda}(1-1) \\
+ & \left.\left(S_{\lambda}^{-}\right)^{2} I_{\lambda}(-1-1)\right] .
\end{aligned}
$$

Designating the angular momenta and magnetic quantum numbers for the initial and final nuclear states by $J_{i} m_{i}$ and $J_{f} m_{f}$, respectively, one has, by the Wigner-Eckart theorem ${ }^{20}$

$$
\begin{aligned}
\int T_{\lambda, \lambda \pm 1}{ }^{-m}(\mathbf{A}, \mathbf{B})=C\left(J_{i} \lambda_{f}\right. & \\
& \left.m_{i}-m\right)\left(J_{f}\left\|T_{\lambda, \lambda \pm 1}\right\| J_{i}\right),
\end{aligned}
$$

where the vector addition coefficient contains all the magnetic quantum number dependence and the remaining factor is a so-called reduced matrix element. The conditions that $\Delta\left(J_{i} \lambda J_{f}\right)$ and $\Delta\left(j j_{\nu} \lambda\right)$ exist express the over-all angular momentum conservation

$$
\mathbf{J}_{i}+\mathbf{J}_{f}=\boldsymbol{\lambda}=\mathbf{j}+\mathbf{j}_{\nu} .
$$

Also $m_{i}=m_{f}+m=m_{f}+\mu+\mu_{\nu}$. For the quantity which should actually appear in the $\beta$-transition probability, one sums (51) over $m_{f}$ and averages over $m_{i}$. Thus, using (52), the pertinent quantity is

$$
\frac{1}{2 J_{i}+1} \sum_{m_{i} m_{f}} I_{\lambda}(11)=\frac{2 J_{f}+1}{2 J_{i}+1}\left|\left(J_{f}\left\|T_{\lambda \lambda+1}\right\| J_{i}\right)\right|^{2}
$$

etc.
As an aid in transcription of notation we append the following useful relations:

$$
\begin{aligned}
T_{0 l}(\mathbf{A}, \mathbf{B}) & =-\frac{3^{\frac{1}{2}}}{4 \pi} \mathbf{A} \cdot \mathbf{B} \delta_{l 1}, \\
T_{\lambda 0^{m}}(\mathbf{A}, \mathbf{B}) & =\frac{3^{\frac{1}{2}}}{4 \pi} B_{m} \delta_{\lambda 1}, \\
T_{11}{ }^{m}(\mathbf{A}, \mathbf{B}) & =-\frac{i}{\sqrt{2}}\left(\frac{3}{4 \pi}\right)(\mathbf{A} \times \mathbf{B})_{m} .
\end{aligned}
$$

For second rank tensors one needs

$$
\begin{aligned}
T_{21} \pm^{2} & (\mathbf{A B})=\frac{3}{4 \pi} A_{ \pm 1} B_{ \pm 1} \\
T_{21}{ }^{ \pm 1}(\mathbf{A B}) & =\frac{1}{\sqrt{2}} \frac{3}{4 \pi}\left(A_{0} B_{ \pm 1}+A_{ \pm 1} B_{0}\right) \\
T_{21}{ }^{0}(\mathbf{A B}) & =\frac{3}{8 \pi}\left(\frac{2}{3}\right)^{\frac{1}{2}}\left(3 A_{0} B_{0}-\mathbf{A} \cdot \mathbf{B}\right) .
\end{aligned}
$$

Thus, for any two operators A, B one has

$$
\begin{gathered}
I_{0}(11)=\frac{3}{16 \pi^{2}}\left|\int \mathbf{A} \cdot \mathbf{B}\right|^{2}, \\
I_{1}(-1-1)=\frac{3}{16 \pi^{2}}\left|\int \mathbf{B}\right|^{2}, \\
I_{1}(00)=\frac{9}{32 \pi^{2}}\left|\int \mathbf{A} \times \mathbf{B}\right|^{2}, \\
I_{2}(-1-1)=\frac{9}{64 \pi^{2}} \sum_{i j}\left|\tau_{i j}(\mathbf{A}, \mathbf{B})\right|^{2} .
\end{gathered}
$$

We now turn our attention to the correction factors for zero rank tensors. For $\lambda=0$ we may write (35) in the simple form ${ }^{21}$

$$
\begin{aligned}
C_{1 P}=\frac{\pi^{2}}{3 p^{2} q^{2} F_{0} M^{2}} \mid \int T_{01}(\mathfrak{r}, \boldsymbol{\sigma}) & \left.\right|^{2} \sum_{\kappa}(2 j+1) \\
& \times\left[\left(f_{\kappa} F_{\kappa}\right)^{\prime}+\left(g_{\kappa} G_{\kappa}\right)^{\prime}\right]_{r=\rho}^{2}
\end{aligned}
$$

since $R_{0}\left(\kappa \kappa_{\nu}\right)=(-)^{l_{\kappa}}(2 j+1)^{\frac{1}{2}} \delta_{\kappa_{\kappa_{\nu}}}=-R_{0}\left(-\kappa,-\kappa_{\nu}\right)$. In (58) the prime denotes $d / d r$. The change in the correction factor, as previously noted, is directly traceable to the appearance of these derivatives.

The result (58) expresses the correction factor in the most compact form and the retardation expansion can

\footnotetext{
${ }^{21}$ Note that

$W(a b c d ; O f)=(-)^{b+c-f} \delta_{a b} \delta_{c d}\left[(2 b+1)(2 c+1) 7^{-1}\right.$.
} 
be made by taking only the terms $\mathrm{s}^{22} \kappa= \pm 1$. Consistently, one replaces $j_{l}(x)$ by the first term in its series expansion; $j_{l}(x) \rightarrow x^{l}[(2 l+1) ! !]^{-1}$. We carry out this operation and in addition we use (37) to replace $f^{\prime}, g^{\prime}$ in terms of $f$ and $g$. In this way the correction factor is expressed in terms of well-tabulated quantities. ${ }^{23}$ These $\operatorname{are}^{14}$

$$
\begin{aligned}
L_{k-1} & =\left(2 p^{2} F_{0}\right)^{-1} \rho^{2-2 k}\left(g_{-k}^{2}+f_{k}^{2}\right), \\
M_{k-1} & =\left(2 p^{2} F_{0}\right)^{-1} \rho^{-2 k}\left(g_{k}^{2}+f_{-k}^{2}\right), \\
N_{k-1} & =\left(2 p^{2} F_{0}\right)^{-1} \rho^{1-2 k}\left(f_{-k} g_{-k}-f_{k} g_{k}\right), \\
P_{k-1} & =\left(2 p^{2} F_{0}\right)^{-1} \rho^{2-2 k}\left(g_{-k}{ }^{2}-f_{k}^{2}\right), \\
Q_{k-1} & =\left(2 p^{2} F_{0}\right)^{-1} \rho^{-2 k}\left(g_{k}^{2}-f_{-k}^{2}\right), \\
R_{k-1} & =\left(2 p^{2} F_{0}\right)^{-1} \rho^{1-2 k}\left(f_{-k} g_{-k}+f_{k} g_{k}\right) .
\end{aligned}
$$

Thus, $L_{0}, M_{0}$, etc., are exactly the same quantities which these symbols have hitherto denoted. Using (55), the correction factor becomes

$$
\begin{aligned}
C_{1 P}=\frac{1}{4 M^{2}} & \left\{\left(U^{2}+1\right) M_{0}-2 U Q_{0}\right. \\
& +\frac{2}{3} q\left[\left(U^{2}-1\right) N_{0}+4\left(U M_{0}-Q_{0}\right)\right] \\
& +\frac{q^{2}}{9}\left[\left(U^{2}+1\right) L_{0}-2 U P_{0}+16 M_{0}\right. \\
& \left.\left.+8\left(U N_{0}-R_{0}\right)\right]\right\}\left|\int \boldsymbol{\sigma} \cdot \mathbf{r}\right|^{2} .
\end{aligned}
$$

Here $U=W-V_{c}-q$ and for most cases $U \approx-V_{c}$. In (60) the term $U^{2} M_{0}$ is dominant while the terms $U Q_{0}$, $U M_{0}$, and $U^{2} N_{0}$ each make about a ten percent correction for $\alpha Z / \rho W_{0} \gg 1$. The remaining terms contribute one percent or less. This statement is borne out by a numerical calculation. This calculation also shows that the coefficient of $|\boldsymbol{S} \boldsymbol{\sigma} \cdot \mathbf{r}|^{2}$ in $C_{1 P}\left(K_{1 P}\right.$, say) is a monotonically increasing function of energy and that for $\mathrm{RaE}$ it rises by 35 percent over the spectrum. This is in contrast to $L_{0}$, which represents the shape of the hitherto used correction factor. Over the range of energies corresponding to the $\mathrm{RaE}$ spectrum $L_{0}$ increases monotonically by 4 percent.

Proceeding in exactly the same manner as described above, we find the correction factors for $P-X$ mixtures. For the $T$ interaction the only zero rank tensor with parity change, yes, is clearly $T_{01}(\mathfrak{r}, \boldsymbol{\sigma})$ or $\boldsymbol{\sigma} \cdot \mathfrak{r}$. From

${ }^{22}$ However, to check the $Z=0$ limit it is necessary to take $\kappa= \pm 2$ in those terms which vanish for $|\kappa|=1$. This peculiarity of the pseudoscalar factor is responsible for the appearance of a correction factor which has the energy dependence characteristic of third forbidden for $Z=0$.

${ }^{23}$ Rose, Perry, and Dismuke, Oak Ridge National Laboratory Report ORNL-1459 (unpublished). These tables are for Coulomb field wave functions. Corrections for finite nuclear size have been given by M. E. Rose and D. K. Holmes, Phys. Rev. 83, 190 (1951) and Oak Ridge National Laboratory Report ORNL-1022 (unpublished).
$T_{\lambda l}(\mathfrak{r}, \boldsymbol{\sigma} \times \mathbf{p})$ the only zero rank tensor is $\mathfrak{r} \cdot(\boldsymbol{\sigma} \times \mathbf{p})$ which has even parity. The interference term is now

$$
\begin{aligned}
C_{1 P T}=-\frac{1}{M}\left\{U M_{0}-Q_{0}+\frac{2 q}{3}\left(2 M_{0}+U N_{0}\right)\right. \\
\left.+\frac{q^{2}}{9}\left(U L_{0}-P_{0}+4 N_{0}\right)\right\}\left|\int \boldsymbol{\sigma} \cdot \mathbf{r}\right|^{2} .
\end{aligned}
$$

For the $A$ interaction, which is given for the sake of completeness, both $\boldsymbol{\sigma} \cdot \mathbf{r}$ and $\boldsymbol{\sigma} \cdot \mathbf{p}$ are possible with the specified rank and parity, and the correction factor for the $A-P$ mixture is

$$
\begin{aligned}
& C_{1 P A}=\frac{1}{M}\left\{-U Q_{0}+M_{0}-\frac{2 q}{3}\left(2 Q_{0}+N_{0}\right)\right. \\
&+\left.\frac{q^{2}}{9}\left(L_{0}-U P_{0}-4 R_{0}\right)\right\}\left|\int \mathbf{\sigma} \cdot \mathbf{r}\right|^{2} \\
&+ \frac{3^{\frac{1}{2}}}{M^{2}}\left\{U R_{0}+N_{0}+\frac{q}{3}\left[U P_{0}-L_{0}+4 R_{0}\right.\right. \\
&\left.\left.-\rho^{2}\left(U Q_{0}-M_{0}\right)\right]-\frac{q^{2} \rho^{2}}{9}\left(4 Q_{0}-U R_{0}+N_{0}\right)\right\} \\
& \quad \times i \int \mathbf{\sigma} \cdot \mathbf{r}\left(\int \mathbf{\sigma} \cdot \mathbf{p}\right)^{*} .
\end{aligned}
$$

From the discussion given in Sec. III, it follows that both matrix element products are of the same order of magnitude. However, for $\alpha Z / \rho \gg 1$ the part of the correction factor associated with the second matrix element product is relatively unimportant.

To these mixture terms one must add the pure $T$ and $A$ contributions $^{1}$

$$
C_{1 T}=\left|\int \boldsymbol{\sigma} \cdot \mathbf{r}\right|^{2}\left(\frac{1}{9} q^{2} L_{0}+M_{0}+\frac{2}{3} q N_{0}\right),
$$

and

$$
\begin{gathered}
C_{1 A}=\left|\int \boldsymbol{\sigma} \cdot \mathbf{r}\right|^{2}\left(\frac{1}{9} q^{2} L_{0}+M_{0}-\frac{2}{3} q N_{0}\right)+\frac{1}{M^{2}}\left|\int \boldsymbol{\sigma} \cdot \mathbf{p}\right|^{2} L_{0} \\
-\frac{2 i}{M} \int \boldsymbol{\sigma} \cdot \mathbf{r}\left(\int \boldsymbol{\sigma} \cdot \mathbf{p}\right)^{*}\left(\frac{1}{3} q L_{0}-N_{0}\right) .
\end{gathered}
$$

In (62) and (64) we have used the fact that the cross product of matrix elements of $\boldsymbol{\sigma} \cdot \mathbf{r}$ and $\boldsymbol{\sigma} \cdot \mathbf{p}$, when multiplied with $i$, is real. ${ }^{24}$

${ }^{24} \mathrm{By}$ application of the time-reversal operator $T$, one can show that all the cross products of matrix elements (with the factors of $i$ where they appear) are real; see C. L. Longmire and A. M. L. Messiah, Phys. Rev. 83, 464 (1951) for a special case. See also L. C. Biedenharn and M. E. Rose, reference 18 . 


\section{APPLICATION TO THE RaE SPECTRUM}

The correction factor for a $T-P$ mixture is given by (60), (61), and (63) in the form

$$
C=g_{P}^{2} C_{1 P}+g_{P} g_{T} C_{1 P T}+g_{T}{ }^{2} C_{1 T} .
$$

Using this correction factor we have attempted to fit the $\mathrm{RaE}$ spectrum, wherein comparison is made with the data of Langer and Price ${ }^{25}$ and of Neary. ${ }^{26}$ The observed spectrum $N(W)$ is compared with $\lambda N_{0}(W) C$ where $N_{0}$ is the allowed shape, $\lambda$ is a scale factor and $C$ is given by $\mathrm{Eq}$. (65). In minimizing

$$
\epsilon^{2}=\sum\left[N(W)-\lambda N_{0}(W) C\right]^{2}
$$

where the sum is over the experimental points $(1.20 \leqslant W$ $\leqslant 2.97)$, the parameters $\lambda$ and $g_{P} / g_{T}$ are available as adjustable quantities. The best fit gives $\epsilon=0.14$ $\times\left[\sum N^{2}(W)\right]^{\frac{1}{2}}$. A relative root-mean-square error of 14 percent implies that the spectrum cannot be fitted with a $T-P$ mixture.

The conclusion to which this result leads is that the spin of RaE is not zero but unity. That this is not an isolated item of evidence leading to this conclusion can be seen from the following considerations.

(a) If the RaE spin is unity it should be possible to fit the spectrum with an $S-T$, or, less likely, ${ }^{5}$ with a $V-T$ mixture. Actually, Yamada ${ }^{27}$ has obtained a fit of the spectrum with an $S-T$ mixture.

(b) The existence of a group of highly favored first forbidden transitions $(\log f t=5-6)$ between nuclei near the doubly magic $82-126$ shell seems to point strongly to the rule that such transitions are characterized by $\Delta J=0$. On the other hand transitions in similar nuclei with $|\Delta J|=1$ show normal first forbidden $f t$ values about 100 times larger. Examples of the first group together with $\log f t$ values and the most plausible assignment of orbitals are: $\mathrm{Tl}^{207} \rightarrow \mathrm{Pb}^{207}(5.16) s_{\frac{1}{2}} \rightarrow p_{\frac{1}{2}} ; \mathrm{Pb}^{209} \rightarrow$ $\mathrm{Bi}^{209}(5.59) g_{9 / 2} \rightarrow h_{9 / 2} ; \quad \mathrm{Tl}^{206} \rightarrow \mathrm{Pb}^{206}(5.18) s_{\frac{1}{2}}$ proton, $p_{\frac{1}{2}}$ neutron coupling to spin 0 in $\mathrm{Tl}^{206} ; \mathrm{Hg}^{205} \rightarrow \mathrm{Tl}^{205}(5.52) p_{\frac{1}{2}}$ $\rightarrow s_{\frac{1}{2}}$. On the other hand for the second group one may cite $\mathrm{Pb}^{205} \rightarrow \mathrm{Tl}^{205}$ (large $\left.f t\right)^{28} \quad p_{\frac{3}{3}} \rightarrow s_{\frac{1}{2}}$; and several other transitions between slightly lighter nuclei; e.g., $\mathrm{Au}^{199} \rightarrow$

${ }^{25}$ I. M. Langer and H. C. Price, Jr., Phys. Rev. 76, 461 (1949). ${ }^{26}$ G. J. Neary, Proc. Roy. Soc. (London) A175, 71 (1940).

${ }^{27}$ M. Yamada, Progr. Theoret. Phys. (Japan) 10, 245 (1953).

${ }^{28}$ C. D. Coryell, quoted by A. de-Shalit and M. Goldhaber, Phys. Rev. 92, 1211 (1953).
$\operatorname{Hg}^{199}(7.73) d_{\frac{3}{2}} \rightarrow p_{\frac{1}{2}} ; \mathrm{Hf}^{181} \rightarrow \mathrm{Ta}^{181}(7.2)^{29} p_{\frac{3}{3}} \rightarrow d_{\frac{5}{3}}$. The decay scheme $^{30}$ of RaE shows that the spins of the 46.5kev state and ground state of $\mathrm{RaE}$ should be different; i.e., either 0 and 1 , respectively, or 1 and 0 . The $\beta$ transition from the $0^{+}$state of $\mathrm{RaD}$ with a $\log f t \sim 5-6$ and the 5-day transition from the ground state with $\log f t=8$ both fit into the above if and only if the $\mathrm{RaE}$ spin is unity. The transitions $\mathrm{Pb}^{212} \rightarrow \mathrm{Bi}^{212^{*}}(5.17) 0^{+} \rightarrow 0^{-}$and $\mathrm{Bi}^{212} \longrightarrow \mathrm{Po}^{212}(7.22) 1^{-}$ $\rightarrow 0^{+}$also fit into the same scheme for similar reasons.

(c) The optical spectrum of RaE as investigated by Fred ${ }^{31}$ shows that the $\lambda 3067$ line of $\mathrm{Bi}^{210}$ shows no hfs splitting. The conclusion from this, that the magnetic moment of the $\mathrm{RaE}$ ground state $<0.18$ nuclear magneton, is at least consistent with a spin 1 assignment ( $h_{9 / 2}$ proton, $g_{9 / 2}$ neutron orbitals).

It is of interest to note that a natural explanation of the favored $\Delta J=0$ first forbidden transitions in heavy elements is afforded by the interaction as described above. From Eq. (60) the correction factor is fairly accurately represented by

$$
g_{P}^{2} C_{1 P} \approx(\alpha Z / 2 \rho)^{4}\left(g_{P}^{2} / M^{2}\right) .
$$

The strong $Z$ dependence exhibited by the $P$ interaction contribution which would enhance transitions of the aforementioned type, could be cited as evidence for the existence of the $P$ interaction. However, in order that the $P$ contribution be comparable with that arising from the $T$ interaction, it is necessary that $g_{P} / g_{T} \sim 50$. It should be emphasized that this conclusion is not a necessary one and if $g_{P} / g_{T} \ll 50$, which would require an independent explanation of the favored first forbidden transitions, one would conclude that the $P$ interaction plays no practical role in the $\beta$ interaction.

\section{ACKNOWLEDGMENTS}

It is a pleasure to record our thanks to Dr. Z. V. Chraplyvy, Dr. M. Fred, Dr. A. de-Shalit, Dr. M. Goldhaber, Dr. R. W. King, and Dr. M. Yamada for making the results of their research available to us before publication. We are indebted to Dr. O. KofoedHansen and Dr. A. Winther for correspondence.

${ }^{29}$ This distinction between $\Delta J=0$ and $|\Delta J|=1$ transitions has already been noted by L. W. Nordheim (Indiana Conference on the Shell Model) and by R. W. King.

${ }^{30} \mathrm{Wu}$, Boehm, and Nagel, Phys. Rev. 91, 319 (1953).

${ }^{31}$ Fred, Tomkins, and Barnes, Phys. Rev. 92, 1324 (1953), and private communication from M. Fred. 
Comparison of the Reactions $p+d \rightarrow \mathrm{H}^{3}+\pi^{+}, p+d \rightarrow$ $\mathrm{He}^{3}+\pi^{0}$ as a Test of Charge Independence, Kenneth C. Bandtel, Wilson J. Frank, and Burton J. Moyer [Phys. Rev. 106, 802 (1957)]. We are indebted to Dr. Sigurd Köhler of CERN for bringing to our attention an error in the reduction of our data. The final ratio of the $\left(t, \pi^{+}\right)$to $\left(\mathrm{He}^{3}, \pi^{0}\right)$ cross sections is raised from $2.3 \pm 0.3$ to $2.6 \pm 0.35$. The ratio of 2 predicted by assuming charge independence is now two standard deviations from the measured value.

In recalculating the ratio of the cross sections we used the latest mass values for pions. Because only 88.7-Mev total energy is available in the center-ofmass system for these reactions, the kinematics are sensitive to small mass differences such as the $\pi^{+}-\pi^{0}$ mass difference. The bombarding proton energy was taken to be exactly $340 \mathrm{Mev}$.

\begin{tabular}{lrcc} 
Particle & Mass (Mev) & Particle & Mass (Mev) \\
\hline proton & 938.23 & $\mathrm{He}^{3}$ & 2808.27 \\
deuteron & 1875.53 & $\pi^{+}$ & 139.63 \\
triton & 2808.80 & $\pi^{0}$ & 135.04
\end{tabular}

The heavy particle was observed at $10.5^{\circ}$ in the laboratory. The newly calculated angles and ratios are now

$$
\begin{array}{ll}
\pi^{+}, 50.1^{\circ} ; & \mathrm{H}^{3}, 129.9^{\circ} ; \\
\pi^{0}, 47.7^{\circ} ; & \mathrm{He}^{3}, 132.3^{\circ} .
\end{array}
$$

$R_{1}=($ the raw experimental data $)=2.86( \pm 11 \%)$,

$$
\begin{aligned}
R_{2} & =\frac{d \sigma}{d \Omega_{\mathrm{c} . \mathrm{m} .}}\left(\pi^{+}, 47.7^{\circ}\right) / \frac{d \sigma}{d \Omega_{\mathrm{c} . \mathrm{m} .}}-\left(\pi^{+}, 50.1^{\circ}\right) \\
R_{3} & =\frac{d \Omega_{\mathrm{lab}}}{d \Omega_{\mathrm{c} . \mathrm{m} .}}\left(\pi^{+}, 50.1^{\circ}\right) / \frac{d \Omega_{\mathrm{lab}}}{d \Omega_{\mathrm{c} . \mathrm{m} .}}\left(\pi^{0}, 47.7^{\circ}\right)=0.86, \\
R & =R_{1} R_{2} R_{3}=2.6( \pm 14 \%) .
\end{aligned}
$$

Problem of Spin Arrangements in $\mathrm{MnO}$ and Similar Antiferromagnets, F. KEFFER AND W. O'Sullivan [Phys. Rev. 108, 637 (1957)]. We wish to acknowledge a prior publication by Loeb and Goodenough, ${ }^{1}$ parts of which we unknowingly duplicated in parts of our paper. They give the general form for the dipolar anisotropy energy of ordering of the second kind [our Eq. (2)], and they also make a very good estimate of the value of the multiplicative constant. Also Loeb ${ }^{2}$ has independently discovered the proportionality between the dipolar interaction energy and the powder neutron diffraction shape factor, and hence he has also noted that the neutron diffraction data cannot distinguish among the possible minimum-energy dipolar configurations. Loeb and Goodenough present an interesting discussion of other sources of anisotropy, and our general discussion is similar to theirs in a number of points. However, the $\mathrm{MnO}$ uniaxial antiparallel spin arrangement which they present is quite different from the Kaplan ${ }^{3}$ arrangement which formed the basis of our model.

${ }^{1}$ Arthur L. Loeb and John B. Goodenough, Proceedings of the Conference on Magnetism and Magnetic Materials, Boston, October, 1956 (American Institute of Electrical Engineers, New York, 1957), pp. 55-68.

2 A. L. Loeb, Congress of International Union of Crystallography, Montreal, July 10-19, 1957; published in Acta Cryst. 10, 780 (1957).

${ }^{3}$ J. I. Kaplan, J. Chem. Phys. 22, 1709 (1954).

Circular Polarization of Bremsstrahlung from Polarized Electrons in Born Approximation, KIRK W. McVoy [Phys. Rev. 106, 828 (1957)]. Figure 1, giving the circular polarization of bremsstrahlung emitted in the forward direction, is incorrect and should be replaced by the figure presented herewith. The degree of polarization is seen to rise much less rapidly with photon energy than was indicated in

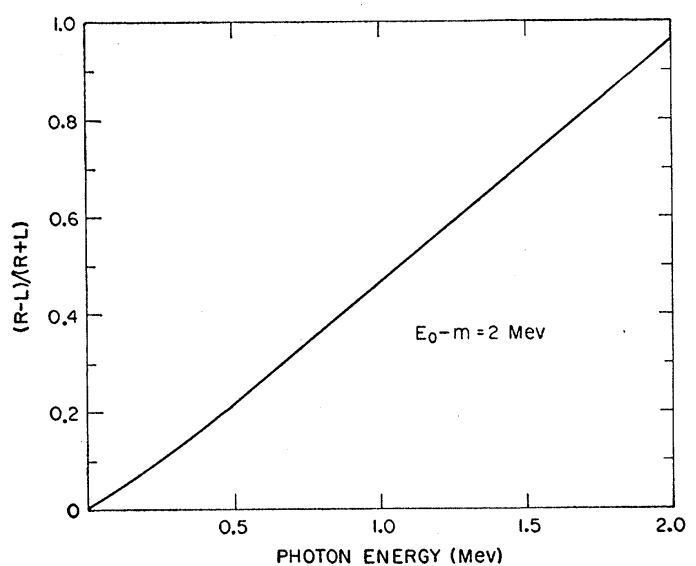

Fig. 1. Circular polarization of the forward bremsstrahlung from 2-Mev "spin-forward" electrons.

the earlier figure; this appears to agree somewhat better with the experimental curve (Fig. 2, lower part) of Goldhaber, Grodzins, and Sunyar. ${ }^{1}$

The error was due to an incorrect sign which arose in the angular integration, and I wish to express my thanks to Dr. C. Fronsdal and Dr. H. Überall for pointing it out to me. It should be noted that this error did not affect any of the expressions [including Eq. (8)] given in the Letter.

${ }^{1}$ Goldhaber, Grodzins, and Sunyar, Phys. Rev. 106, 826 (1957).

Pseudoscalar Interaction and the Beta Spectrum of RaE, M. E. Rose AND R. K. Osborn [Phys. Rev. 93, 1315 (1954)]. In Eq. (62), the factor $3^{\frac{1}{2}}$ which appears as a coefficient of the $A P$ interference term should be deleted. 
Comparison of the Reactions $p+d \rightarrow \mathrm{H}^{3}+\pi^{+}, p+d \rightarrow$ $\mathrm{He}^{3}+\pi^{0}$ as a Test of Charge Independence, Kenneth C. Bandtel, Wilson J. Frank, and Burton J. Moyer [Phys. Rev. 106, 802 (1957)]. We are indebted to Dr. Sigurd Köhler of CERN for bringing to our attention an error in the reduction of our data. The final ratio of the $\left(t, \pi^{+}\right)$to $\left(\mathrm{He}^{3}, \pi^{0}\right)$ cross sections is raised from $2.3 \pm 0.3$ to $2.6 \pm 0.35$. The ratio of 2 predicted by assuming charge independence is now two standard deviations from the measured value.

In recalculating the ratio of the cross sections we used the latest mass values for pions. Because only 88.7-Mev total energy is available in the center-ofmass system for these reactions, the kinematics are sensitive to small mass differences such as the $\pi^{+}-\pi^{0}$ mass difference. The bombarding proton energy was taken to be exactly $340 \mathrm{Mev}$.

\begin{tabular}{lrcc} 
Particle & Mass (Mev) & Particle & Mass (Mev) \\
\hline proton & 938.23 & $\mathrm{He}^{3}$ & 2808.27 \\
deuteron & 1875.53 & $\pi^{+}$ & 139.63 \\
triton & 2808.80 & $\pi^{0}$ & 135.04
\end{tabular}

The heavy particle was observed at $10.5^{\circ}$ in the laboratory. The newly calculated angles and ratios are now

$$
\begin{array}{ll}
\pi^{+}, 50.1^{\circ} ; & \mathrm{H}^{3}, 129.9^{\circ} ; \\
\pi^{0}, 47.7^{\circ} ; & \mathrm{He}^{3}, 132.3^{\circ} .
\end{array}
$$

$R_{1}=($ the raw experimental data $)=2.86( \pm 11 \%)$,

$$
\begin{aligned}
R_{2} & =\frac{d \sigma}{d \Omega_{\mathrm{c} . \mathrm{m} .}}\left(\pi^{+}, 47.7^{\circ}\right) / \frac{d \sigma}{d \Omega_{\mathrm{c} . \mathrm{m} .}}-\left(\pi^{+}, 50.1^{\circ}\right) \\
R_{3} & =\frac{d \Omega_{\mathrm{lab}}}{d \Omega_{\mathrm{c} . \mathrm{m} .}}\left(\pi^{+}, 50.1^{\circ}\right) / \frac{d \Omega_{\mathrm{lab}}}{d \Omega_{\mathrm{c} . \mathrm{m} .}}\left(\pi^{0}, 47.7^{\circ}\right)=0.86, \\
R & =R_{1} R_{2} R_{3}=2.6( \pm 14 \%) .
\end{aligned}
$$

Problem of Spin Arrangements in $\mathrm{MnO}$ and Similar Antiferromagnets, F. KEFFER AND W. O'Sullivan [Phys. Rev. 108, 637 (1957)]. We wish to acknowledge a prior publication by Loeb and Goodenough, ${ }^{1}$ parts of which we unknowingly duplicated in parts of our paper. They give the general form for the dipolar anisotropy energy of ordering of the second kind [our Eq. (2)], and they also make a very good estimate of the value of the multiplicative constant. Also Loeb ${ }^{2}$ has independently discovered the proportionality between the dipolar interaction energy and the powder neutron diffraction shape factor, and hence he has also noted that the neutron diffraction data cannot distinguish among the possible minimum-energy dipolar configurations. Loeb and Goodenough present an interesting discussion of other sources of anisotropy, and our general discussion is similar to theirs in a number of points. However, the $\mathrm{MnO}$ uniaxial antiparallel spin arrangement which they present is quite different from the Kaplan ${ }^{3}$ arrangement which formed the basis of our model.

${ }^{1}$ Arthur L. Loeb and John B. Goodenough, Proceedings of the Conference on Magnetism and Magnetic Materials, Boston, October, 1956 (American Institute of Electrical Engineers, New York, 1957), pp. 55-68.

2 A. L. Loeb, Congress of International Union of Crystallography, Montreal, July 10-19, 1957; published in Acta Cryst. 10, 780 (1957).

${ }^{3}$ J. I. Kaplan, J. Chem. Phys. 22, 1709 (1954).

Circular Polarization of Bremsstrahlung from Polarized Electrons in Born Approximation, KIRK W. McVoy [Phys. Rev. 106, 828 (1957)]. Figure 1, giving the circular polarization of bremsstrahlung emitted in the forward direction, is incorrect and should be replaced by the figure presented herewith. The degree of polarization is seen to rise much less rapidly with photon energy than was indicated in

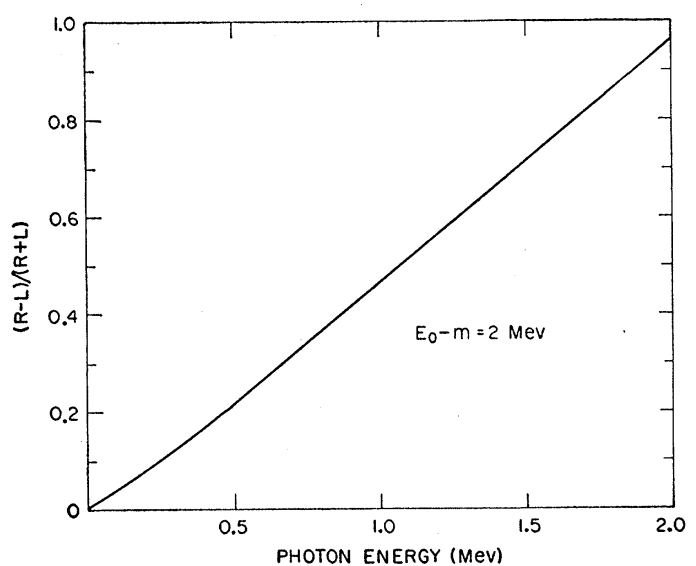

Fig. 1. Circular polarization of the forward bremsstrahlung from 2-Mev "spin-forward" electrons.

the earlier figure; this appears to agree somewhat better with the experimental curve (Fig. 2, lower part) of Goldhaber, Grodzins, and Sunyar. ${ }^{1}$

The error was due to an incorrect sign which arose in the angular integration, and I wish to express my thanks to Dr. C. Fronsdal and Dr. H. Überall for pointing it out to me. It should be noted that this error did not affect any of the expressions [including Eq. (8)] given in the Letter.

${ }^{1}$ Goldhaber, Grodzins, and Sunyar, Phys. Rev. 106, 826 (1957).

Pseudoscalar Interaction and the Beta Spectrum of RaE, M. E. Rose AND R. K. Osborn [Phys. Rev. 93, 1315 (1954)]. In Eq. (62), the factor $3^{\frac{1}{2}}$ which appears as a coefficient of the $A P$ interference term should be deleted. 